\title{
LOCAL LOW PRESSURE AREAS IN ANTICLINE STRUCTURES
}

\author{
Boris P. Sibiryakov ${ }^{1}$, Lourenildo W.B. Leite ${ }^{1}$, Egor P. Sibiryakov² and Wildney W.S. Vieira ${ }^{1}$
}

\begin{abstract}
In order to localize low pressures zones in sedimentary basins for oil and gas exploration, it is necessary to know $\mathrm{P}$ and $\mathrm{S}$ wave velocities for medium. Strictly speaking, we need to know the rock densities for all layers, and in addition there are many correlation tables between seismic velocities and densities; besides, density is a parameter admitted to change slowly with depth to the top of the target interface. $P$ wave velocities are considered a conventional asset, and $S$ wave velocities can be obtained from special field survey, in particular from converted P-S waves registered by VSP technology, and by petrophysical measurements. The theory in this paper deals with stress prediction in the subsurface, and takes in consideration the constitutive parameters (density and Lame's), and the geometry of the reservoir target surface. The model does not separate the different contributions (porosity, fluids) to the rock velocities controlled by the constitutive parameters. It is not a necessary condition that an anticline be a potential structure for oil and gas accumulation. This role can be played by horizontal structures if there is a positive $\gamma=\frac{V_{S}}{V_{P}}$ ratio discontinuity, or a negative discontinuity of the Poisson, $\sigma$, ratio across the horizontal boundary. These conditions are responsible for producing a pressure discontinuity, such that beneath the boundary there will be a sufficiently lower pressure zone than above the boundary. In this case, the lower horizontal boundary is said to be an attractor surface for fluids of the any kind; in the opposite case, this boundary does not have fluid attractor properties.
\end{abstract}

Keywords: seismic structured media, porous media, anticline structures, pressure prediction.

RESUMO. Com o objetivo de localizar zonas de baixa pressão em bacias sedimentares voltadas à exploração de óleo e gás, é necessário conhecer as velocidades das ondas P e S para o meio. Mais especificamente, precisamos conhecer a densidade das rochas em todas as camadas, e aditivamente existem várias tabelas de correlação entre velocidade e densidade das rochas; além disso, é um parâmetro que varia lentamente com a profundidade até o topo da interface-alvo. A velocidade das ondas Pé considerada uma informação convencional, e a velocidade das ondas S pode ser obtida por levantamentos especiais de campo, em particular a partir da conversão P-S registrada por tecnologia VSP, e por medidas petrofísicas. A teoria deste trabalho trata da predição de tensão na subsuperfície, e leva em consideração os parâmetros constitutivos (densidade e de Lamé), e a topografia em superfície do reservatório-alvo. 0 modelo não separa as diferentes contribuições (porosidade e fluidos) para estabelecer as velocidades nas rochas controladas pelos parâmetros constitutivos. Não é uma condição necessária que uma superfície anticlinal seja uma estrutura potencial para o acúmulo de óleo e gás. Esta condição pode ser representada por uma superfície horizontal, se existir uma discontinuidade na razão $\gamma=\frac{V_{S}}{V_{P}}$, ou uma descontinuidade negativa na razão de Poisson, $\sigma$, através da superfície. Estas condições são responsáveis por produzir uma descontinuidade de pressão, de tal forma que abaixo da interface existirá uma zona de pressão mais baixa do que há acima da mesma. Neste caso, a parte inferior da superfície é considerada como um atrativo de fluidos de qualquer tipo; e no caso oposto, esta superfície não é dotada de propriedades de atração de fluidos.

Palavras-chave: meios sísmicos estruturados, meios porosos, meios fraturados.

\footnotetext{
1 Federal University of Pará, Campus Universitário do Guamá, Instituto de Geociências, 66075-110 Belém, PA, Brazil. Phone: +55(91) 3201-7693; Fax: +55(91) 32017609 - E-mails: Iwbleite@gmail.com; SibiryakovBP@gmail.com; wildneyvieira@gmail.com

${ }^{2}$ Russian Academy of Sciences, Siberian Branch, Trofimuk Geology and Geophysics Petroleum Institute - E-mail: SibiryakovEP@gmail.com
} 


\section{INTRODUCTION}

The anticline structure can be a very useful trap, especially if it has a negative discontinuity in the $\gamma=\frac{V_{S}}{V_{P}}$ ratio. In this case, exists also an additional horizontal stretching due to the negative curvature of the anticline structure. It is interesting, that the effects of slope and curvature are in opposite directions; the slopes produce an additional compression, while the average curvature produces a horizontal stretching.

If there is an anticline structure with a positive discontinuity in the $\gamma$ ratio, it can be a compensational effect. The additional pressure due to the $\gamma$ discontinuity, and the additional stretching due to the average curvature may eliminate each other. In this case, the anticline structure is not a fluid attractor.

The present paper is part of a major project under the theme prediction of stresses and strains using $\mathrm{P}$ and $\mathrm{S}$ wave velocities in order to localize areas of low pressure in oil and gas productive layers as natural suction pumps. This project is structured in different and independent parts, and as a result the paper Sibiryakov et al. (2013b) is already accepted for publication, and another by Sibiryakov et al. (2013a) has been submitted and is under review.

The first part of the project is related to conventional seismic investigations in order to obtain the distribution of the $\mathrm{P}$ and $S$ wave velocities, and also to obtain the configuration of seismic boundaries in sedimentary basins. The second part is related to the prediction of stress and strain in the geological structures, and also to the prediction of the nontrivial behavior of pressure, since it can decrease with depth and create natural pumps that accumulate fluids. The third part is related to the prediction of discontinuity in pressure between solid and fluid, what depends on the structure of pore space.

In the present description we restrict our attention to isotropic models, and for anisotropic situations the equations are more complicated, there are more control parameters, and the data needs more processing. Every layer forming the geological 3D structure model has constant elastic parameters.

It is mandatory that the acquired data be three components, otherwise it is necessary to apply special processing to obtain the $S$ wave information from P-S phase conversion. $S$ waves can be used from land data obtained with horizontal vibroseis and VSP technology, and from marine data using AVO technology looking for converted P-S-P waves. In special cases, we can use petrophysical measurements of borehole samples for $V_{P}$ and $V_{S}$ and density $\rho$.

The first published appearances about pore space and integral geometry were presented by Sibiryakov (2002) and Sibiryakov \& Prilous (2007). The theory of porous media is based on integral geometry, because such mathematical discipline deals with collective geometrical properties of real reservoirs. It has been shown by Santaló (1953) that such collective properties are namely for porosity, specific surface area, Average curvature and Gaussian curvature (Smirnov, 1964). For example, cracked media have as a rule small porosity, but very large specific surface area, what creates anomalous high $\gamma$ ratio, and it means that the Poisson coefficient, $\sigma=\frac{1-2 \gamma^{2}}{2-2 \gamma^{2}}$, can be negative, and this discussion can be seen in Sibiryakov (2013) and Sibiryakov \& Sibiryakov (2010).

\section{METHODOLOGY}

\section{The role of slope angles and curvatures}

In order to predict the stress-strain state in geological structures we need to integrate the elastic equations of equilibrium. The boundary conditions are for continuity of forces and displacements. The equilibrium equations contain the elastic parameters; that is, the $V_{P}$ and $V_{S}$ velocities, and the rock densities.

It should be clear that these parameters and the boundary configuration have to be obtained from the seismic processing and imaging. It means that we need to have detailed velocity analysis from previous investigations. As for the special case of the shear wave velocity distribution, results of exploration with special explosion and horizontal vibroseis can be used.

The stresses in geological structures represent a very complicate subject in a six dimensional space, because there are in the usual case six components of the stress tensor in any point of the medium.

The present work is dedicated to the solution of a simpler problem: the pressure prediction in the vicinity of geologic structural boundaries. The scalar invariant pressure is very important, and it is the simplest characteristic of stress-strain condition. This paper answers a question about the boundary attraction for fluids, or the condition of a boundary not being a fluid attraction.

Figure 1 represents a model for a sedimentary basin, where we aim at a reservoir volume limited on the top by a $S$ surface, where the layers above it are responsible for the overload weight that causes the stress field in the underground rocks. The stress pattern varies according to the $\gamma$ ratio, that can present important discontinuities across the interfaces. Therefore, the aim is the $S$ surface where the stress discontinuity will varie according to its topographic form, and this effect measured by the spatial slopes and curvatures of the reference $S$ surface. The physical aspects of this theory does not take geological faulting and lithological variations in the rock volume, and only the bending of the formations (above and below the $S$ interface) that defines the anticline 
structure. For geological representations, special block drawings for reservoir representations are found, for instance, in Chopra \& Marfurt (2007).

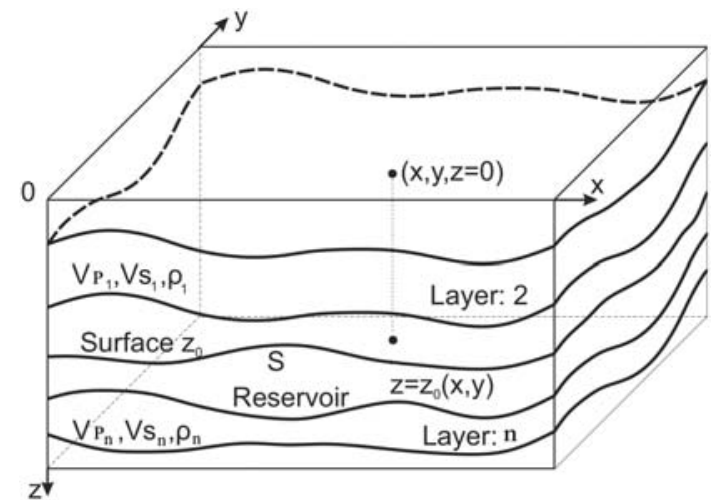

Figure 1 - Block perspective illustrating a sedimentary basin. It shows the Cartesian arbitrary system $(x, y, z)$, the layer blocks limited by curved interfaces, a subtle reservoir volume limited above by the $S$ surface represented by $z=z_{0}(x, y)$, and a flat free surface at $z=0$.

In the usual case, a geological structure represents a very complicate problem for the solution of the equilibrium equations, which are given by (Kupradze, 1963; Novacky, 1975):

$$
\begin{aligned}
& \frac{\partial \sigma_{x x}}{\partial x}+\frac{\partial \sigma_{y x}}{\partial y}+\frac{\partial \sigma_{z x}}{\partial z}=0 ; \\
& \frac{\partial \sigma_{x y}}{\partial x}+\frac{\partial \sigma_{y y}}{\partial y}+\frac{\partial \sigma_{z y}}{\partial z}=0 ; \\
& \frac{\partial \sigma_{x z}}{\partial x}+\frac{\partial \sigma_{y z}}{\partial y}+\frac{\partial \sigma_{z z}}{\partial z}=\rho g ;
\end{aligned}
$$

where the symbology and units are: $\sigma\left[\mathrm{N} / \mathrm{m}^{2}\right]$ for stress, $\rho\left[\mathrm{kg} / \mathrm{m}^{3}\right]$ for density, and $g\left[\mathrm{~m} / \mathrm{s}^{2}\right]$ for gravity acceleration. The spatial variables $(x, y, z)$ stand for the Cartesian system of coordinates, with $z$ pointing positive downwards inside the underground. Physically, the system of Eqs. (1) means that: (1st) the sum of the stress variation along the vertical axis is given by the weight of the overburden column; (2nd) the sum of the stress variation along the horizontal $x$-axis is chosen to be null; and (3rd) the sum of the stress variation along the $y$-axis is also chosen to be null. The gravity acceleration, $g=g(z)$, is considered constant in the underground volume in consideration, and also $g=g_{z}$ when it is needed a convenient notation.

The total solution of the system of Eqs. (1), $u^{(\mathrm{T})}=u^{(\mathrm{C})}+u^{(\mathrm{P})}$, is given by the complementary solution, $u^{(\mathrm{C})}$, of the three homogeneous equations, added to the particular solution, $u^{(P)}$, of the inhomogeneous system obtained via Green's function and convolution (Roach, 1986).

The particular solution for the displacement component $u_{k}(\mathbf{x}),(k=x, y, z)$, is given by the Poisson integral with respect to the structural volume $V$ as:

$$
u_{k}(\mathbf{x})=g \frac{1}{V_{s}^{2}} \int_{V} \Gamma_{k z}(\mathbf{x}, \mathbf{y}) d V_{\mathbf{y}} .
$$

It is interesting that this integral depends mainly on the shear velocity $V_{S}\left[L T^{-1}\right] . \Gamma_{k z}(\mathbf{x}, \mathbf{y})\left[L^{-1}\right]$ is the Green tensor for the system of Eqs. (1) (fundamental solution, where in the third equation $\rho g$ is replaced by $\rho g \delta(x) \delta(y) \delta(z))$, and it is given by Kupradze (1963).

For the solution represented by Eq. (2), and others in the sequel based on this formulation (see, for instance, Eqs. (17) and (31)), once the displacement field, $u_{k}(\mathbf{x})$, is known, then the deformation, stress, and pressure fields can be calculated. But, we demonstrate ahead for simple models that the contribution of the particular solution in Eq. (2) is small and, as a result, the complementary solution is more important.

The system of Eqs. (1) with the particular solution in Eq. (2), and a possible general complementary solution, establish a very complicate problem. To obtain a complementary solution, $u^{(\mathrm{C})}$, is already a special problem by itself.

However, we can obtain an elegant complementary solution to system of Eqs. (1) by considering a plausible model described by simple geometric relations for the $S$ surface, $z=z_{0}(x, y)$, and by the overburden weight components $P_{k}=\rho g z n_{k}$, $\left[\mathrm{N} / \mathrm{m}^{2}\right]$, in the form:

$$
\begin{aligned}
& P_{x}(S)=\rho g z_{0}(x, y) n_{x} \\
& P_{y}(S)=\rho g z_{0}(x, y) n_{y} \\
& P_{z}(S)=\rho g z_{0}(x, y)
\end{aligned}
$$

where $n_{i}=\cos \left(n, x_{i}\right)$ is the direction cosine between the surface normal vector, $\vec{n}$, and the arbitrary $(x, y, z)$ Cartesian system. The stress expressions for Eqs. (3) (with $\sigma$ for normal and $\tau$ tangential stress components) on the interface $S$ are written as:

$$
\begin{aligned}
P_{x}(S) & =\sigma_{x x} n_{x}+\tau_{y x} n_{y}+\left.\tau_{z x} n_{z}\right|_{S} \\
& =\rho g z_{0}(x, y) n_{x} ; \\
P_{y}(S) & =\sigma_{y y} n_{y}+\tau_{x y} n_{x}+\left.\tau_{z y} n_{z}\right|_{S} \\
& =\rho g z_{0}(x, y) n_{y} ; \\
P_{z}(S) & =\sigma_{z z} n_{z}+\tau_{x z} n_{x}+\left.\tau_{y z} n_{y}\right|_{S} \\
& =\rho g z_{0}(x, y) .
\end{aligned}
$$

We can now consider that the rock displacements on the boundary $z=z_{0}(x, y)$ to be related with the vertical displacement 
by the formulas:

$$
\begin{gathered}
u_{x}=u_{z} \cos (\rho g, x) \\
u_{y}=u_{z} \cos (\rho g, y) \\
\cos (\rho g, z)=1
\end{gathered}
$$

On the boundary, represented by the surface $z=z_{0}(x, y)$, the vertical strain is given by the relation:

$$
e_{z z}=\frac{\partial u_{z}}{\partial z}=\frac{\rho g z(x, y)}{\lambda+2 \mu} ;
$$

that comes from Eqs. (1) considering a flat structure. Under integration, Eq. (6) gives the displacement,

$$
\begin{aligned}
u_{z}\left(z_{0}(x, y)\right) & =\int_{0}^{z_{0}} \frac{\rho g z(x, y)}{\lambda+2 \mu} d z \\
& =\frac{\rho g z_{0}^{2}(x, y)}{2(\lambda+2 \mu)} .
\end{aligned}
$$

In the above equation, the quantities $\rho$ and $g$ are allowed to varie as a function of $z(x, y)$; but, the solution in the right hand consider them constant with $z(x, y)$.

The horizontal strain, $e_{x x}=\frac{\partial u_{x}}{\partial x}=u_{x, x}$, with Eq. (5), using the convenient symbology, is expressed by:

$$
e_{x x}=\frac{\partial}{\partial x} u_{z}(x, y) \cos (n, x) ;
$$

with the result under derivation by parts,

$$
\begin{gathered}
e_{x x}=u_{z, x} \frac{z_{0, x}}{\sqrt{1+z_{0, x}^{2}+z_{0, y}^{2}}} \\
-u_{z} \frac{z_{0, x x}}{\sqrt{1+z_{0, x}^{2}+z_{0, y}^{2}}}\left(1-\frac{z_{0, x}^{2}}{1+z_{0, x}^{2}+z_{0, y}^{2}}\right) .
\end{gathered}
$$

For the above Eq. (9),

$$
u_{z, x}(x, y)=\frac{\rho g}{\lambda+2 \mu} z_{0}(x, y) \frac{\partial z_{0}(x, y)}{\partial x} .
$$

Equation (9), with Eq. (7), can be rewritten in the following form:

$$
\begin{gathered}
e_{x x}\left(z_{0}(x, y)\right)=\frac{\rho g z_{0}}{\lambda+2 \mu} \frac{z_{0, x}^{2}}{\sqrt{1+z_{0, x}^{2}+z_{0, y}^{2}}} \\
-\frac{\rho g z_{0}^{2}}{2(\lambda+2 \mu)} \frac{z_{0, x x}}{\sqrt{1+z_{0, x}^{2}+z_{0, y}^{2}}} \\
\times\left(1-\frac{z_{0, x}^{2}}{1+z_{0, x}^{2}+z_{0, y}^{2}}\right) .
\end{gathered}
$$

The total dilatation $(\theta=\nabla \cdot \vec{u})$ (vertical compression and horizontal decompression) on the boundary $z=z_{0}(x, y)$ takes the result:

$$
\begin{gathered}
\theta\left(z_{0}(x, y)\right)=\frac{g z_{0}}{V_{P}^{2}}\left[1+\frac{z_{0, x}^{2}+z_{0, y}^{2}}{\sqrt{1+z_{0, x}^{2}+z_{0, y}^{2}}}\right] \\
-\frac{g z_{0}^{2}}{2 V_{P}^{2}}\left[z_{0, x x} \varphi_{1}(x, y)+z_{0, y y} \varphi_{2}(x, y)\right]
\end{gathered}
$$

where,

$$
\begin{aligned}
& \varphi_{1}(x, y)=\frac{1+z_{0, y}^{2}}{\left(1+z_{0, x}^{2}+z_{0, y}^{2}\right)^{3 / 2}} ; \quad \text { and } \\
& \varphi_{2}(x, y)=\frac{1+z_{0, x}^{2}}{\left(1+z_{0, x}^{2}+z_{0, y}^{2}\right)^{3 / 2}} .
\end{aligned}
$$

The quantity named Pressure $P$ is defined as the average of the normal stresses; that is:

$$
P=\frac{1}{3}\left(\sigma_{x x}+\sigma_{y y}+\sigma_{z z}\right) ;
$$

and it is the first invariant of the stress tensor. Using the generalized Hooke's law for isotropic medium:

$$
\sigma_{i j}=\lambda \theta \delta_{i j}+2 \mu e_{i j} ;
$$

the pressure in Eq. (14) is now directly related to the dilatation, and we specify it in the form,

$$
P_{\theta}=\left(\lambda+\frac{2}{3} \mu\right) \theta=K \theta,
$$

where $K=\lambda+\frac{2}{3} \mu$ stands for the pressure module.

Some observations about the dilatation Eq. (12) are now important.

First, that Eq. (12) depends on $V_{P}$, and on the first and second order space derivatives of the surface $z=z_{0}(x, y)$. The first derivative terms, $\left(z_{0, x}(x, y), z_{0, y}(x, y)\right)$, are slope angles. The second derivative terms, $\left(z_{0, x x}(x, y), z_{0, y y}(x, y)\right)$, relate to the surface general curvature (Smirnov, 1964).

Second, in the case that the P wave velocity does not change across the boundary (this is very rare situation), the dilatation has a continuous value. But, in the usual case the $\mathrm{P}$ wave has a discontinuity across the boundary and, as given above, the pressure is given by the product of pressure module $(K)$ to the dilatation $(\theta)$, that can also change across the boundary (Sibiryakov et al., 2004).

Third, the first term of the Eq. (12) contains the square of the first derivative, which means that, for not very large angles, the 
slope effect results in the increase of pressure due to the structure. However, the curvature effect is more interesting.

Fourth, for negative curvature (anticline structure) there is a decrease in pressure, and this effect increases with depth due to the $z_{0}^{2}(x, y)$ factor in the second term of Eq. (12), instead of in the first term where there is the factor $z_{0}(x, y)$.

Fifth, and continuing, the sign of the second derivative is negative, and the curvature is also of a negative value. This means, the anticline structure produces a low pressure zone, which is a favorable condition for fluid accumulation.

For positive curvature (sincline structure), we have the opposite effect.

Sixth, consider the ideal case of a spherical arc; then, the value of the second term in Eq. (12) may have the value of the first term. This means that the negative curvature produces a planar stretching near the top and shortening near the rim.

Seventh, the first term in Eq. (12) is related to the slope angles, with a positive contribution to the dilatation. This means that this term produces an increase in compression as a function of the increase in the amplitude of the anticline structure.

The question that we raise now is: When is it possible that the simple representations in Eq. (5) is sufficiently accurate to diminish the pressure field in the vicinity of the anticline dome?

It should also be clear that the contributions of the Poisson integral in Eq. (2) to the displacement and stress fields are small, in comparison to the fields due to elementary geometrical and physical properties of structures (tangent and vertical forces, and displacements along the structure boundary).

\section{Contribution of the Poisson Integral to Displacements}

We can represent the contribution of the Poisson integral Eq. (2) to the displacement field as the difference of two integrals in the form:

$$
\Delta u_{k}(\mathbf{x})=g\left(\frac{1}{V_{S}^{(+) 2}}-\frac{1}{V_{S}^{(-) 2}}\right) \int_{V} \Gamma_{k z}(\mathbf{x}, \mathbf{y}) d V_{\mathbf{y}},
$$

where $V_{S}^{(+)}$(above) and $V_{S}^{(-)}$(below) are seismic wave velocities across the structure $S$ boundary. The integrand is the Green tensor given by:

$$
\begin{gathered}
\Gamma_{k z}(\mathbf{x}, \mathbf{y})=\frac{1}{8 \pi}\left[\left(1+\gamma^{2}\right) \delta_{k z}\right. \\
\left.+\left(1-\gamma^{2}\right) \frac{\left(\mathbf{x}_{k}-\mathbf{y}_{k}\right)\left(z-z^{\prime}\right)}{r^{2}(\mathbf{x}, \mathbf{y})}\right] \frac{1}{r(\mathbf{x}, \mathbf{y})},
\end{gathered}
$$

where $\mathbf{y}=\left(x^{\prime}, y^{\prime}, z^{\prime}\right)$ is the integration variable throughout the volume $V$. The two integrals in Eq. (17) are interpreted as material substitution: the first integral relates to the material which is eliminated from the structure, and the second to the material which is occupied by the real structure. Figure 2 illustrates the coordinate system, the geometry of the reservoir volume $V$, the integration variable $\mathbf{y}$ in the volume $V$, and the reference point $\mathbf{x}$ along the $S$ surface.

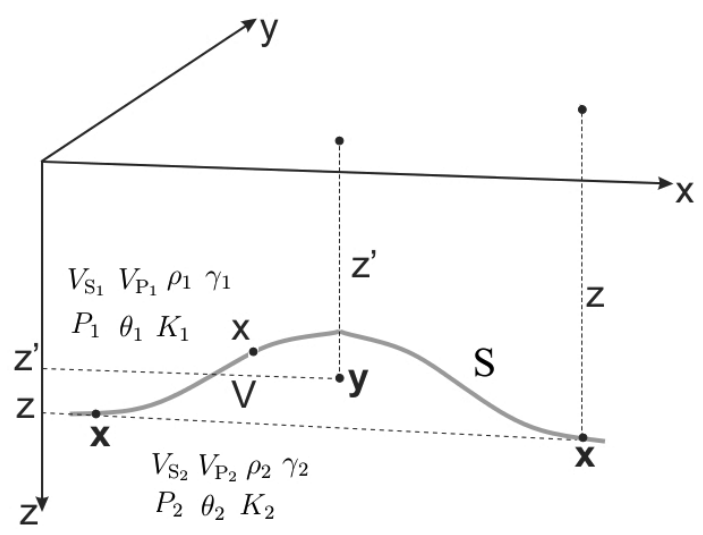

Figure 2 - Block diagram representing a reservoir volume $V$ limited above by the surface $S$ represented by $z=z_{0}(x, y)$. The integration variable $\mathbf{y}$ and the $S$ surface reference point $\mathbf{x}$ are also shown.

The quantity $r$ is the geometrical distance between the $\mathbf{x}$ and $\mathbf{y}$ points. The contribution in Eq. (17) vanishes if the velocities $V_{S}^{(+)}$and $V_{S}^{(-)}$are equal. On the other hand, the displacement field due to the elementary method expressed by Eqs. (6) and (7) is given by:

$$
u_{z}^{0}=\frac{\rho g z_{0}^{2}}{2(\lambda+2 \mu)}=\frac{g z_{0}^{2}}{2 V_{P}^{2}} .
$$

Let us consider a simple but important structure model represented by a spherical body characterized by the volume $V=\pi R^{2} h$, where $R$ is the average radius of the structure, and $h$ is the amplitude. The result for the integral in Eq. (17) gives a simple and good numerical condition for an estimation method (formulas of the type in Eq. (12)), that is given by:

$$
\frac{R h}{8} \frac{\Delta V_{S}}{V_{S}} \ll 4 \gamma^{2} z_{0}^{2}
$$

where $\Delta V_{S}=V_{S}^{(+)}-V_{S}^{(-)}$. Considering that the $\gamma$ ratio be about $\gamma^{2} \approx 0.25$, then the numerical condition in Eq. (20) simplifies to:

$$
\frac{h}{R} \frac{\Delta V_{S}}{V_{S}} \ll 8\left(\frac{z_{0}}{R}\right)^{2} .
$$

This interesting result says that for small value of $h$ with respect to $R$, the Eq. (21) is true, specially for large $z_{0}$, and it establishes that a spherical segment represents well an anticline structure. 


\section{Contribution of the Poisson Integral to Stresses}

For estimating forces $P_{k},(k=x, y, z)$, at the $S$ boundary from stresses $\sigma_{k i}$, such that $P_{k}=\sigma_{k i} n_{i}$, there is an analogous equation to the one for displacement in Eq. (17), which is given by:

$$
\begin{aligned}
& P_{k}(\mathbf{x})=\frac{1}{4 \pi} \rho g_{z} \int_{V} P \Gamma_{k z}(\mathbf{x}, \mathbf{y}) d V_{\mathbf{y}}=-\frac{1}{4 \pi} \rho g_{z} \int_{V} \\
& \quad \times\left\{\left[a \delta_{k z}+b \frac{\left(\mathbf{x}_{k}-\mathbf{y}_{k}\right)\left(z^{\prime}-z\right)}{r^{2}}\right] \frac{\partial}{\partial n}\left(\frac{1}{r}\right)\right. \\
& \left.+a\left[\cos \left(n, \mathbf{x}_{k}\right) \frac{z^{\prime}-z}{r^{3}}-\cos (n, z) \frac{\mathbf{x}_{k}-\mathbf{y}_{k}}{r^{3}}\right]\right\} d V_{\mathbf{y}}
\end{aligned}
$$

where

$$
a=\frac{1}{2 \pi} \frac{\mu}{\lambda+2 \mu}, \quad b=\frac{3}{2 \pi} \frac{\lambda+\mu}{\lambda+2 \mu},
$$

and $P$ under the integral stands for a general operator for the concentrated forces in the integration point $\mathbf{y}$; that means, placed at in the Green function $\Gamma_{k z}$, that includes differentiation, multiplication, summation and convolution to construct the force field (Kupradze, 1963). Comparing $\Gamma_{k z}$ in Eq. (17) with $P \Gamma_{k z}$ in Eq. (22) above, the $P\left[L^{-1}\right]$ operator stands for the directional derivative $\frac{\partial}{\partial n}=n_{k} \frac{\partial}{\partial x_{k}}$ with respect to the normal to the $S$ surface (see Fig. 2).

Considering the structure to be a spherical segment, as done for obtaining the result in Eq. (20) followed by Eq. (21) above, the estimation of stresses for this simple model gives the numerical condition:

$$
\frac{\rho g V}{2 \pi R^{2}}\left(\gamma_{1}^{2}-\gamma_{2}^{2}\right) \ll \rho g z_{0} .
$$

Or, in another simplified form, as:

$$
\frac{h}{2}\left(\gamma_{1}^{2}-\gamma_{2}^{2}\right) \ll z_{0} .
$$

The estimations in Eqs. (24) and (21) mean that the simple spherical model offer a good method for stress estimation, if the depth $z_{0}$ to the structure is much larger than its amplitude $h$; this is a usual situation in most oil deposits.

\section{Test Example: Anticline Structure with Low Slope Angles}

Figure 3 shows the case of an anticline structure modeled by a Gaussian surface defined by:

$$
z_{0}(x, y)=H-h e^{-\left(\frac{x^{2}+y^{2}}{a^{2}}\right)},
$$

where $H$ is the depth to the rim of the structure, $a$ the average radius, and $h$ is the amplitude of the Gaussian dome.

For calculating the pressure across the model surface, the parameters for the two media are defined as: $V_{P}$ above is
$3000 \mathrm{~m} / \mathrm{s}$, and $3200 \mathrm{~m} / \mathrm{s}$ below; $\gamma=V_{S} / V_{P}$ above is 0.5 , and 0.577 below; the density is $\rho=3000 \mathrm{~kg} / \mathrm{m}^{3}$ above and below; and the gravity value was taken as $g=9.8 \mathrm{~m} / \mathrm{s}^{2}$. The figures that follow are the results obtained with these parameter values.

Figure 4 shows the elementary overburden pressure field $P_{0}=P_{z}(S)=\rho g z_{0}$ behavior above the $S$ surface, and with a consistent low around the dome.

Figure 5 shows the overburden weight pressure discontinuity $\Delta P_{0}=\frac{4}{3} P_{0}\left(\gamma_{1}^{2}-\gamma_{2}^{2}\right)$ form across the $S$ surface, and with a consistent low around the dome as expected for the given parameters.

Figure 6 shows the cubic dilatation $\theta$ calculated with Eq. (12), where the red color is for the medium above, and the green color for the medium below the $S$ surface. The figure shows a consistent form for the dilatation with respect to the specified model.

Figure 7 shows the distribution of the dilatation pressure $P_{\theta}$, where is clear a low area around and under the dome. This distribution is calculated by $P_{\theta}=\left(\lambda+\frac{2}{3} \mu\right) \theta=P_{\theta}\left(z_{0}\right)$ using Eq. (12) referenced to the $S$ surface $z_{0}(x, y)$. The pressure immediately above the $S$ surface (blue color) is given by $P_{\theta}^{(+)}=P_{\theta}-\frac{1}{2} \Delta P_{\theta}$, and immediately below (red color) by $P_{\theta}^{(-)}=P_{\theta}+\frac{1}{2} \Delta P_{\theta}$.

The pressure unit used is $\mathrm{N} / \mathrm{m}^{2}=1$ Pascal $(\mathrm{Pa})$, which is equivalent to $1 \mathrm{~Pa}=9.8692 \times 10^{-6}$ atm (atmosphere).

It is interesting to observe that the pressure below the $S$ surface (blue color) is sufficiently less than the pressure above (red color) by about $1.0 \times 10^{7}\left[\mathrm{~N} / \mathrm{m}^{2}\right]$ (about 200 atmospheres). The main role in pressure decrease is played by the negative curvature of the anticline arc, and by the negative discontinuity of the $\gamma$ parameter. This structure acts as an attractor for fluids. Besides, we can see a pressure increase near the periphery of the structure, and this means that around the periphery of an anticline there is a border for fluid migration to below, or to above, the anticline $S$ surface.

Figure 8 shows the pressure discontinuity $\Delta P_{\theta}$ across the $S$ surface, where is clear a low pressure area around and under the dome. This is a convenient figure to see the nuance and details for the analysis of fluid migration around the dome and rim of the structure. Fluids should migrate from a high to low a pressure zone, but we still have to consider the petroleum geology principles to form a complete analysis of the migration process as, for instance, the source and sealing rocks, and structural attitudes.

Figure 9 shows the difference between the pressure discontinuities as calculated by the two related models: overburden minus dilatation pressures, and given by $\Delta P_{0 \theta}=\Delta P_{0}-\Delta P_{\theta}$, using results as shown in Figures 5 and 8 . The rim area shows 


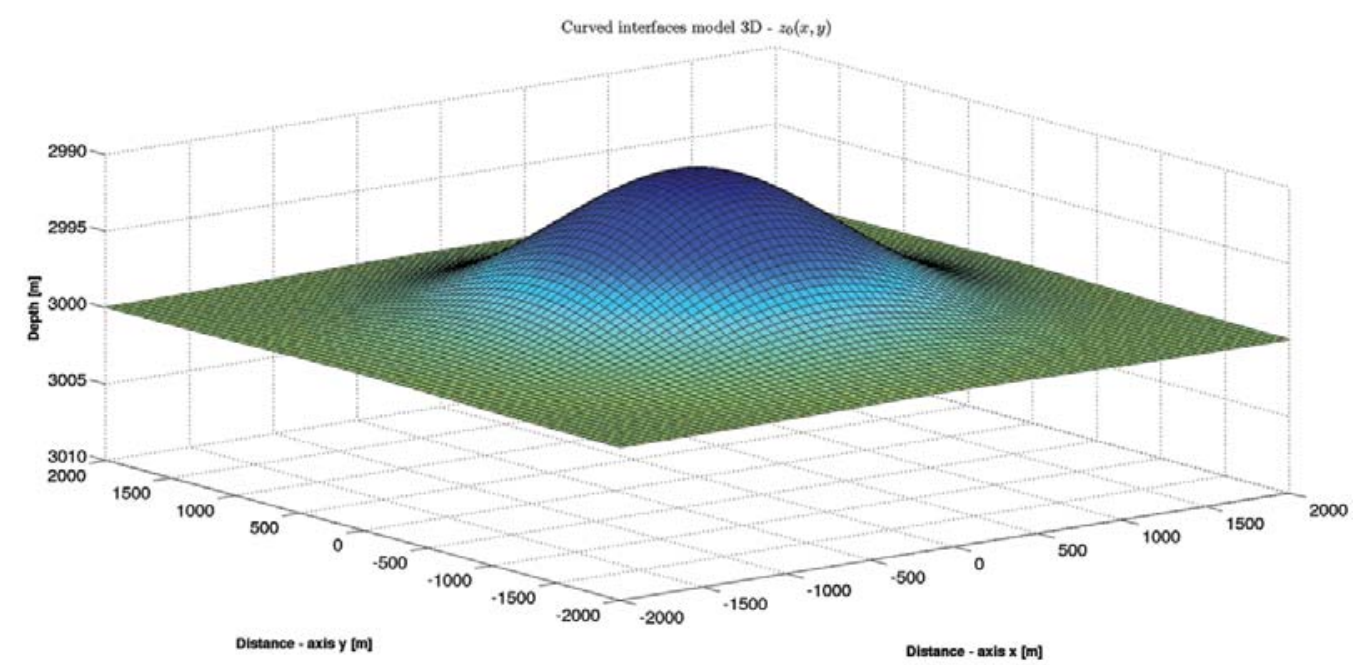

Figure 3 - Topography of the anticline model according to Eq. (25) representing the $S$ surface separating the two media. The vertical axis indicates the surface position and amplitude $z_{0}(x, y)$ for $h=10, H=3000$, and $a=1000$.

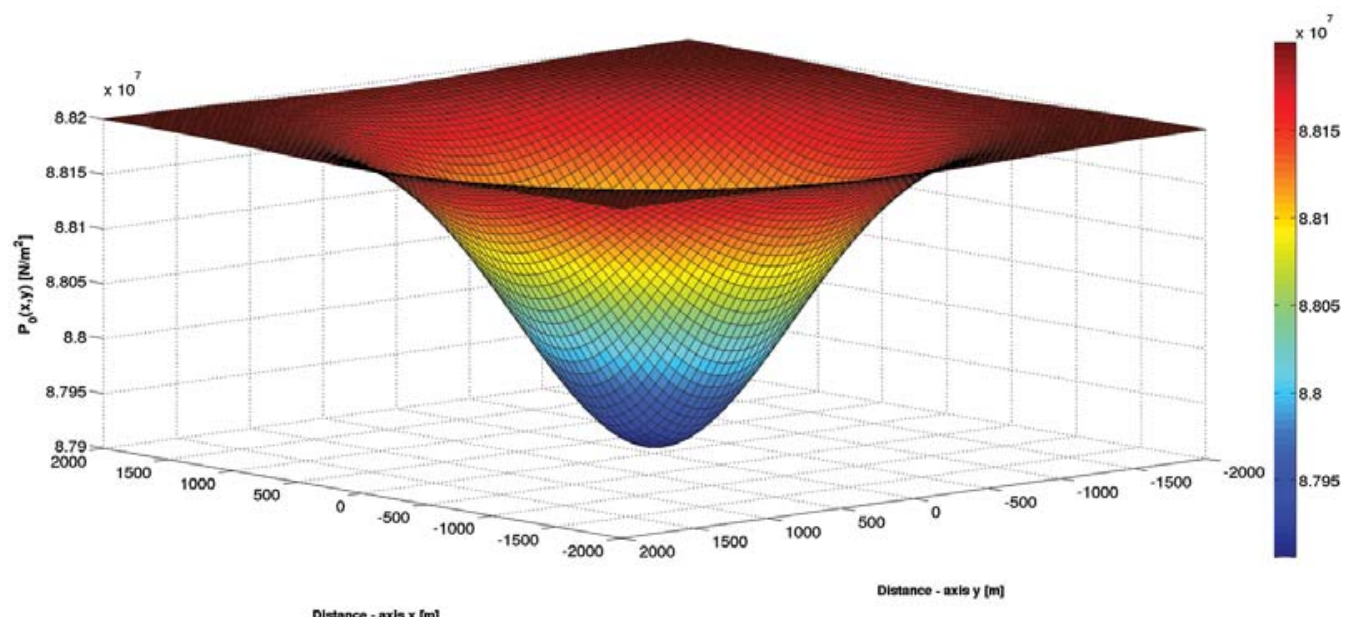

Figure 4 - Normal overburden weight as pressure $P_{0}$ according to Eq. (3).

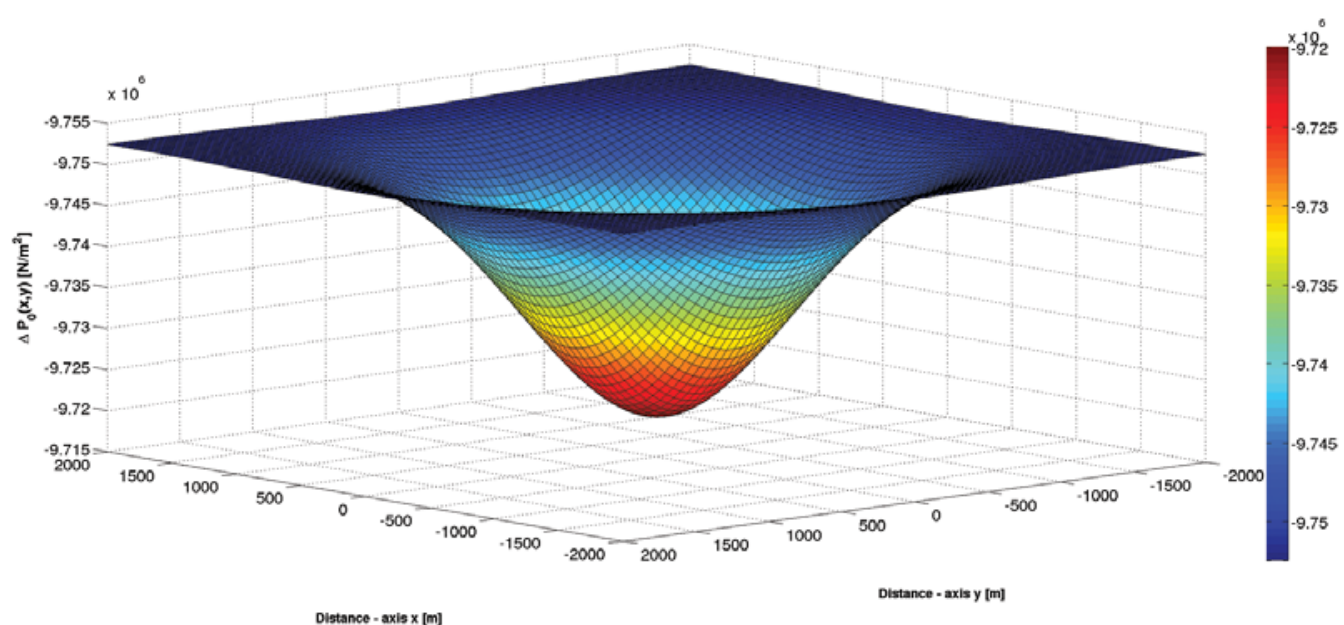

Figure 5 - Normal overburden weight as pressure discontinuity $\Delta P_{0}$ across the $S$ surface, and consistent with the results of Figure 4. 


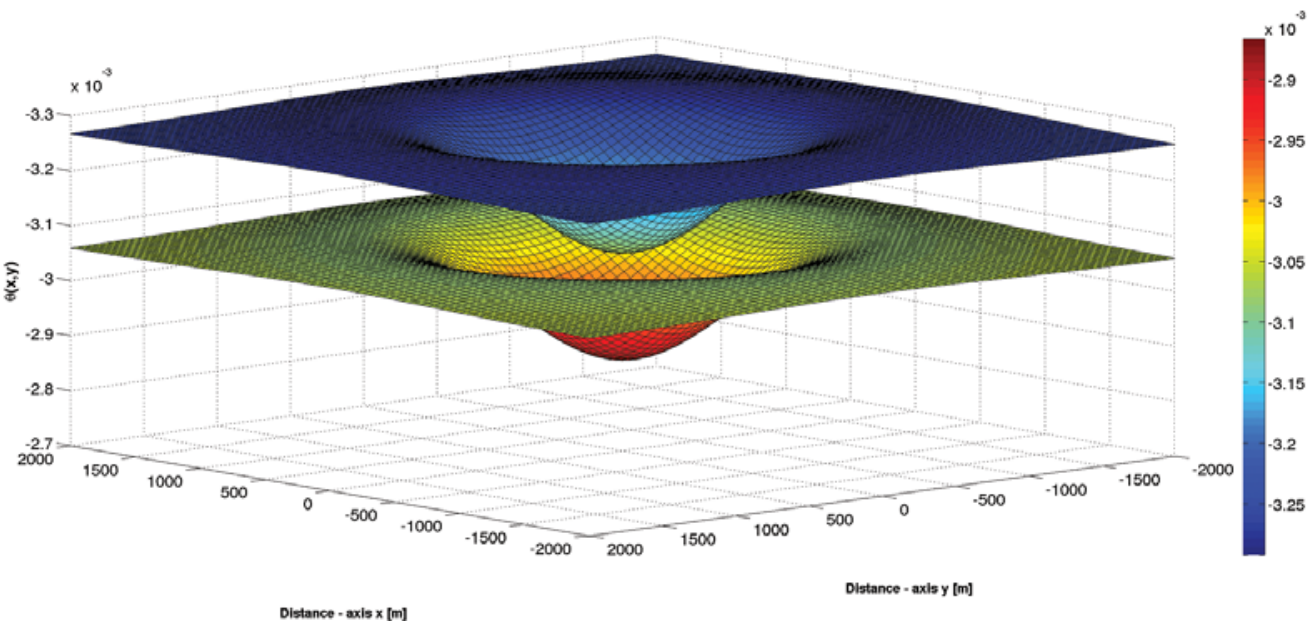

Figure 6 - Cubic dilatation $\theta$ according to Eq. (12). The values in green are for the layer below the $S$ surface, and in blue for the layer above $S$.

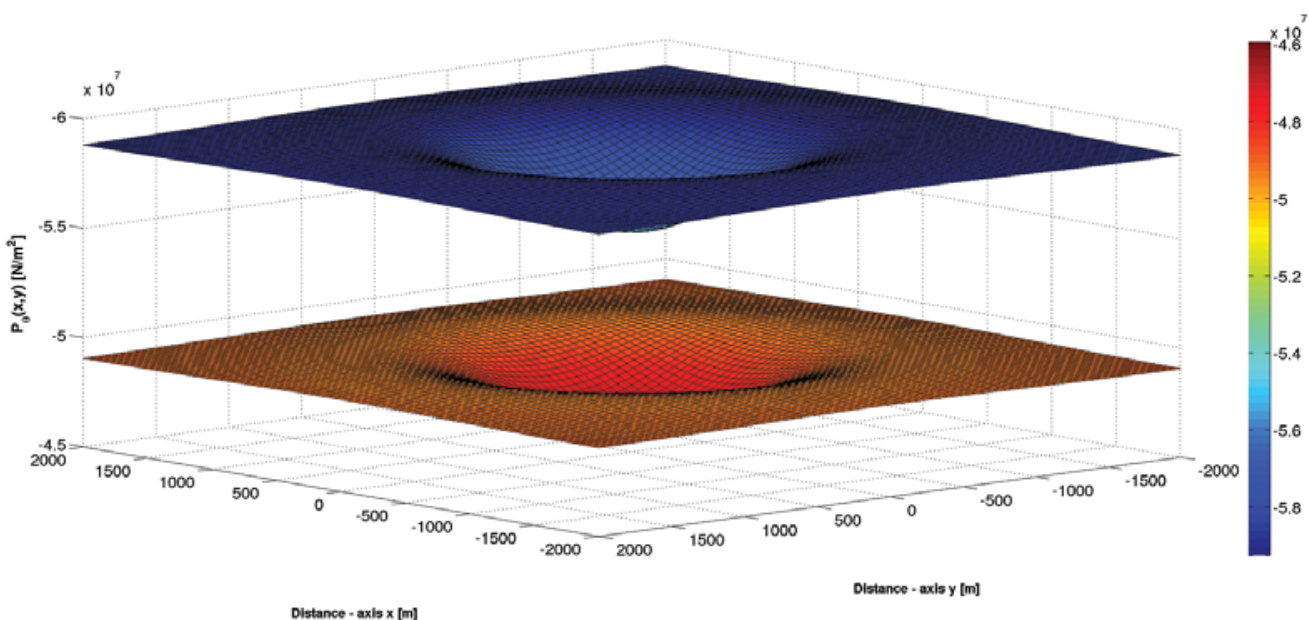

Figure 7 - This structure is a fluid attractor. Result for the dilatation pressure $P_{\theta}$ using Eq. (12) and $\theta$ as shown in Figure 6 . The blue color is for the medium above, and the red color is for the medium below the $S$ surface.

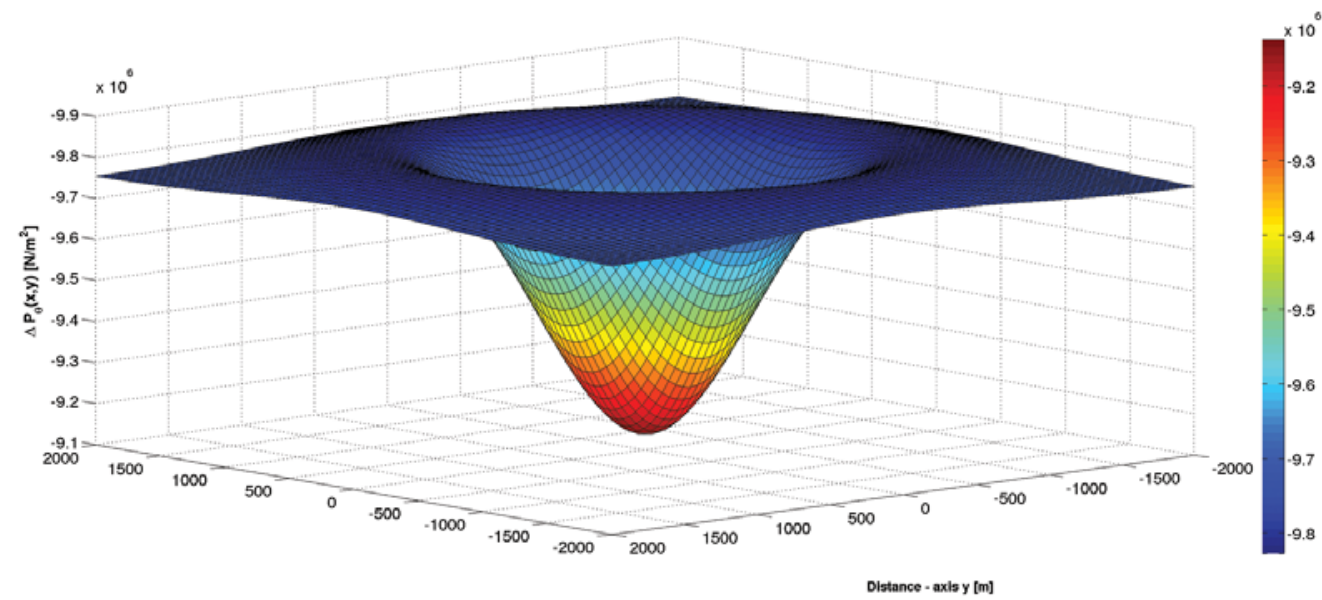

Figure 8 - Result for the dilatation pressure discontinuity $\Delta P_{\theta}=K_{1} \theta_{1}-K_{2} \theta_{2}$ using the results in Figure 7 to analyze the details of the pressure variation around the dome and rim. 


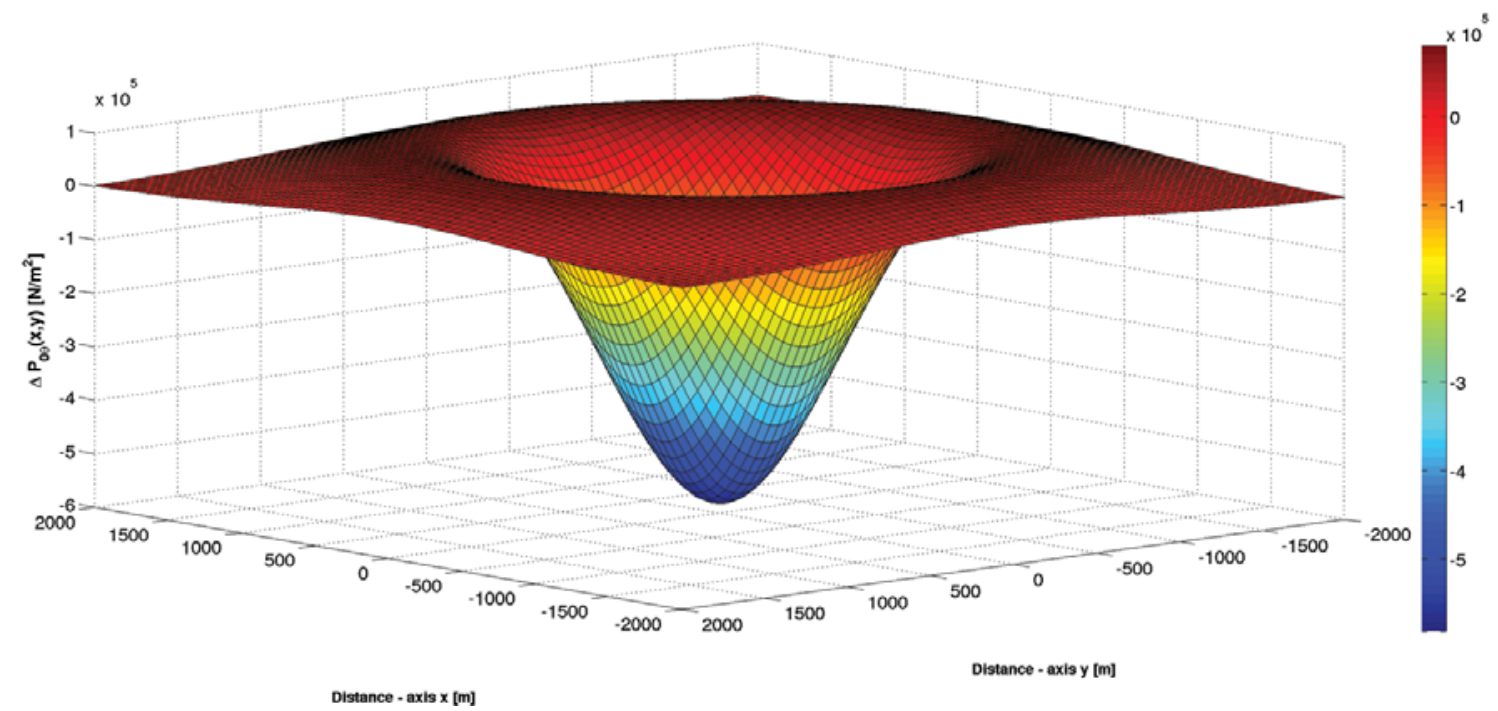

Figure 9 - Difference between the overburden and the dilatation pressure discontinuities $\Delta P_{0 \theta}=\Delta P_{0}-\Delta P_{\theta}$ using the results as shown in Figures 5 and 8.

the expected value around zero, and the dome presents a discrepancy between these two models, but by only around $0.1 \mathrm{~atm}$, what represents a good approximation considering a discontinuity of $200 \mathrm{~atm}$ for $\Delta P_{0}$ and $\Delta P_{\theta}$.

For the case of Figure 10, we inverted the physical conditions through the parameters of the anticline; that is, $\gamma=0.577$ above, and $\gamma=0.5$ below the $S$ surface. The result gives another picture, as the inverse of the Figure 7 ; that is, the pressure below the $S$ surface is larger than above by about $1.0 \times 10^{7}\left[\mathrm{~N} / \mathrm{m}^{2}\right]$ (about 200 atmospheres), and the vicinity of the structure is not a fluid attractor.

\section{Test Example: Anticline Structure with High Slope Angles}

We treat now an application of the modified boundary integral equation method as described by Sibiryakov (2006) for the case of pressure prediction for arbitrary structural forms. In the development of the theory, it is established continuity of the displacement vector $\mathbf{u}$, and of the force vector $\mathbf{P}$ across the boundary surface.

The method corresponds to the solution of the inhomogeneous elastic static equation, considering a model formed by homogeneous layers (the $\lambda, \mu$, and $\rho$ parameters are constant in every layer), which is given by:

$$
\mu \Delta \mathbf{u}_{i}+(\lambda+\mu) \operatorname{grad}_{i} \operatorname{div} \mathbf{u}=-\rho g \mathbf{e}_{z},
$$

where the $\mathbf{e}_{z}$ is the unit vector for the $z$-axis.
We consider a boundary $S$ surface, as shown in Figure 11, represented by the parametrical form:

$$
\left\{\begin{array}{l}
x=r \cos \varphi \\
y=r \sin \varphi \\
z(r)=H+h \exp \left(-\alpha r^{2}\right)
\end{array}\right.
$$

that separates two different media, one above and one below. The distance parameter $r$ changes from 0 to $1 \mathrm{~km}$ with a step of $1 / 200$, $\varphi$ from 0 to $2 \pi$ with a step of $\pi / 100$, the rim at $H=-0.5 \mathrm{~km}$, the height of the dome $h=0.25 \mathrm{~km}$, and the dispersion factor as $\alpha=10$. The upper boundary (free surface) is the plane represented by $z=0$. The parameters of the upper layer are simplified to

$$
\lambda^{(+)}=\mu^{(+)}=\rho^{(+)}=g^{(+)}=1 .
$$

The lower medium has also the same parameters, but $\lambda^{(-)}=$ 0.8 .

The problem is devided in two steps; the first deals with the particular solution, and the second with the complementary solution of Eq. (26).

Step 1: The particular solution of the inhomogeneous Eq. (26), without satisfying boundary conditions, are specified by the following simple expressions. For above the $S$ surface by:

$$
\begin{gathered}
\mathbf{u}^{(+)}=\left(0,0,-\frac{\rho^{(+)} g z^{2}}{2\left(\lambda^{(+)}+2 \mu^{(+)}\right)}\right) \\
\text {at } z \in\left(-z_{0}, 0\right) .
\end{gathered}
$$




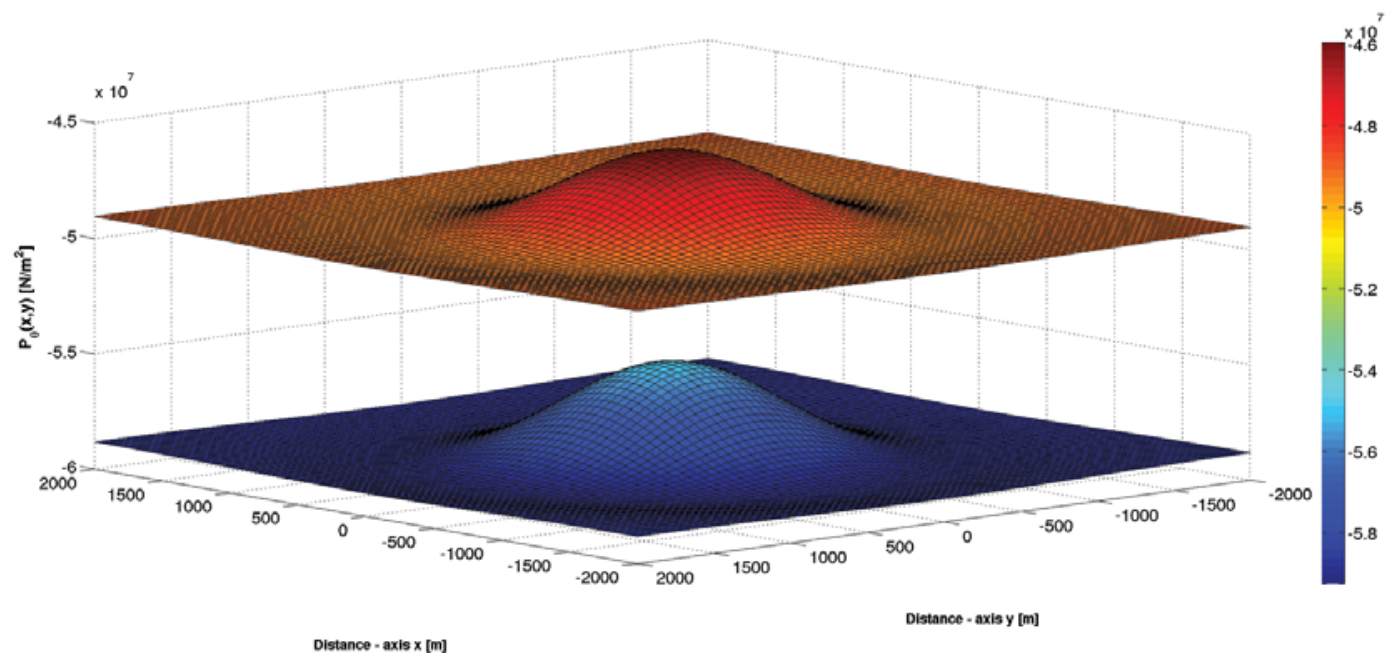

Figure 10 - This structure is not a fluid attractor. The parameters have the inverse values of the ones for the case of Figure 7: blue for $\gamma=0.5$ below, and red for $\gamma=0.577$ above the $S$ surface.

And for below the $S$ surface by:

$$
\begin{gathered}
\mathbf{u}^{(-)}=\left(0,0,-\frac{\rho^{(-)} g\left(z-z_{0}\right)^{2}}{2\left(\lambda^{(-)}+2 \mu^{(-)}\right)}\right. \\
-\frac{\rho^{(+)} g z z_{0}}{\left(\lambda^{(-)}+2 \mu^{(-)}\right)}+\frac{\rho^{(+)} g z_{0}^{2}}{\left(\lambda^{(-)}+2 \mu^{(-)}\right)} \\
\left.-\frac{\rho^{(+)} g z_{0}^{2}}{2\left(\lambda^{(+)}+2 \mu^{(+)}\right)}\right) \quad \text { at } \quad z \in\left(-\infty,-z_{0}\right) .
\end{gathered}
$$

Step 2: The complementary solution of the homogeneous Eq. (26) adds to the particular solutions in Eqs. (28) and (29), and satisfies the boundary conditions on the structure surface and on the free flat surface. Therefore, the starting form is:

$$
\mu \Delta \mathbf{u}_{i}+(\lambda+\mu) \operatorname{grad}_{i} \operatorname{div} \mathbf{u}=0,
$$

with the solution for the displacement component, $u_{i}$, given by

$$
u_{i}(\mathbf{x})=\frac{1}{2 \pi} \int M_{i k}(\mathbf{x}, \mathbf{y}) F_{k}(\mathbf{y}) d S_{\mathbf{y}} .
$$

The force component operator, $P_{i}\left(M_{i k}\right)=P_{i}$, obtained from solution in Eq. (31), satisfies the Fredholm integral equation of the second kind:

$$
P_{i}(\mathbf{x})=F_{i}(\mathbf{x})-\frac{1}{2 \pi} \int P_{i k}(\mathbf{x}, \mathbf{y}) F_{k}(\mathbf{y}) d S_{\mathbf{y}} .
$$

The algorithm for calculating the tensor kernels $M_{i k}(\mathbf{x}, \mathbf{y})$ and $P_{i k}(\mathbf{x}, \mathbf{y})$, from where the vector function $F_{i}(\mathbf{y})$ is obtained, was published in Sibiryakov (2006) where, for the possibility of the tensor calculations, it is necessary to solve a linear algebraic system, where one part is formed by Fredholm equations of the first kind, and the other part by Fredholm equations of the second kind.

For large slope angles (more than 45 degrees), the tangent increases fast, and the approximate Eqs. (11) and (12) are no longer valid.

Figure 11 shows the topography of the anticline structure, that varies with radial distance from the center of the structure. The pressure around this structure is calculated using numerical accurate methods of boundary integral equations, and the details of this method is discussed in the paper by Sibiryakov (2006). This method is used to calculate pressure for any surface geometrical form; but, for the present example we used a Gaussian dome characterized by steep side slopes.

Figure 12 represents further calculations over the case of Figure 11 to show details of the pressure variation, where the values outside the dome are approximately as given by Eqs. (11) and (12). But, here we call attention to fact of the predictive oscillating behavior of pressure around the sides of the structure, that can be important for the analysis of fluid migration.

\section{CONCLUSIONS}

Zones of low pressure exist not only in anticline structures; but, they can also be present in horizontal layers if the $\gamma$ ratio is smaller in the layer above than in the layer below with respect to the structure surface. The search for such zones requires the knowledge of both $\mathrm{P}$ and $\mathrm{S}$ seismic velocity distributions, which 


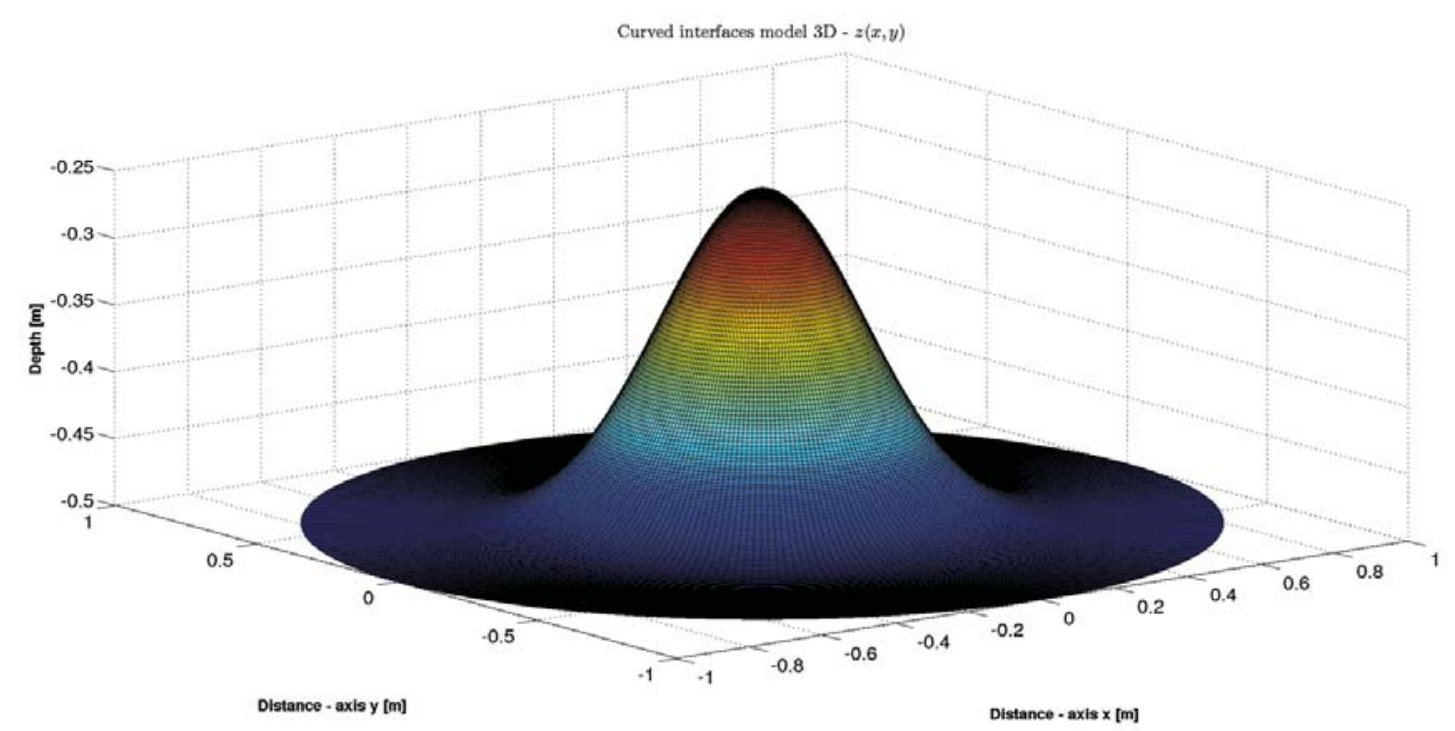

Figure 11 - Anticline surface topography according to Eq. (27). Case of a structure with steep slope angles. The color scale informs the ordinate $z(r)$ values.

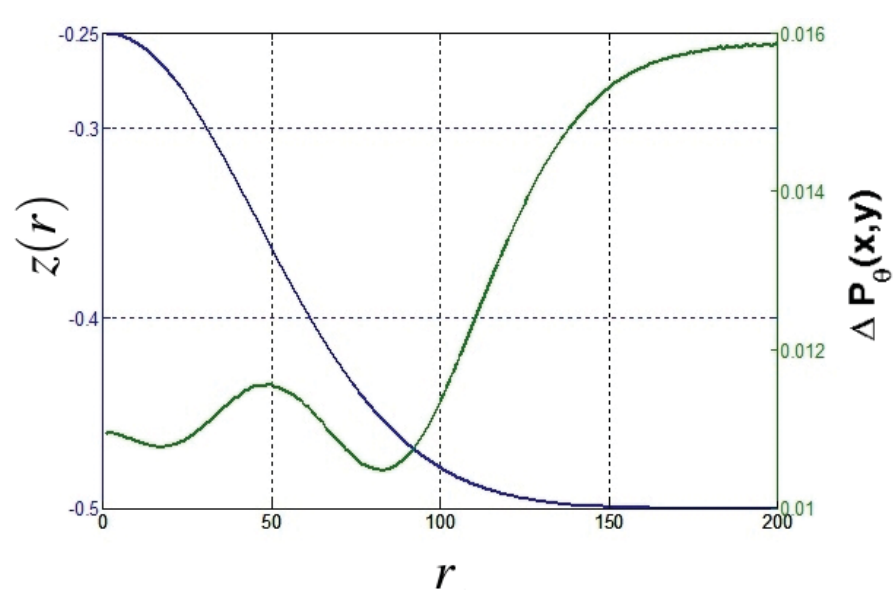

Figure 12 - The vertical left axis gives the structural Gaussian anticline topography. The vertical right axis gives the pressure discontinuity $\Delta P_{\theta}$ versus radial distance from the dome central part. The green line shows a special oscillating pressure variation details, and an increase towards outside the dome. The right vertical axis had the sign changed for simplifying the reading.

can be determined by seismic processing, VSP and laboratory measurements.

The local decrease of pressure near the dome of an anticline structure depends on the discontinuity of the physical parameters across the structural surface, and on the geometrical parameters (slope angle and curvature). The quantity physically affected is the stress field, and the constitutive parameters (density, Lame's, and if needed the porosity, specific surface area, etc.) are admitted constant for the volume rock under the static condition. The volume rocks that form the anticline extend laterally to a horizontal attitude with the same constitutive parameter values.
The negative discontinuity of pressure causes the decrease of pressure below the structure surface, which turns it an attractor for fluid accumulation.

The positive discontinuity of pressure causes an increase of pressure below the structure surface, and as a result this structure is not an attractor feature for fluid accumulation.

The role of structural curvature is to increase its effect on the pressure value as a function of depth of the structure; that means, as the depth increases the role of the curvature also increases.

Figures 9 and 12 serve also as a numerical sensitivity analysis of the model considering the effect of the anticline dome to 
the pressure discontinuity across the target interface, that may serve as a natural suction pump for fluids (gas, water and oil) in the migration process.

\section{ACKNOWLEDGEMENTS}

The authors would like first to thank the Reviewers for their patience and positive contribution. We would like to thank the Brazilian institutions UFPA (Universidade Federal do Pará), FINEP (Financiadora de Estudos e Projetos), Petrobras (Petróleo Brasileiro S/A), and to the project National Institute of Science and Technology (Instituto Nacional de Ciência e Tecnologia, INCT-GP, of MCT/CNPq/FINEP) for the research support aiming at oil exploration. The thanks are also extended to CAPES for the scholarship. Special thanks go to the Project Science Without Borders of CAPES/CNPq of the Brazilian Federal Government that sponsors a main part of this research work developed at UFPA/IG/CPGF.

\section{REFERENCES}

CHOPRA S \& MARFURT KJ. 2007. Seismic attributes for prospect identification and reservoir characterization. Tulsa, USA: Society of Exploration Geophysicists, 464 pp.

KUPRADZE VD. 1963. The potential method in elasticity. Moscow: Physics and Mathematics Issue, 472 pp.

NOVACKY V. 1975. Theory of Elasticity. Moscow: Mir, 872 pp.

ROACH GF. 1986. Green's Functions. London: Cambridge University Press, $325 \mathrm{pp}$.
SANTALÓ L. 1953. Introduction to integral geometry. Hermann et Cie. Editors, Paris, France, 123 pp.

SIBIRYAKOV BP. 2002. Supersonic and intersonic cracking in rock-like material under remote stresses. Theoretical and Applied Fracture Mechanics, 38(3): 255-265.

SIBIRYAKOV EP. 2006. On a boundary integral equation method used to determine parameters of microheterogeneus media (in Russian). Physical Mesomechanics, 9: 97-101.

SIBIRYAKOV BP \& PRILOUS BI. 2007. The unusual small wave velocities in structured bodies and instability of pore or cracked media by small vibration. WSEAS Transactions on Applied and Theoretical Mechanics, 2(7): 139-144.

SIBIRYAKOV EP \& SIBIRYAKOV BP. 2010. The structure of pore space and disjoining pressure in granular medium. Physical Mesomechanics, 13(Special Issue): 40-43.

SIBIRYAKOV BP, SIBIRYAKOV EP, GLEBOV AF, NESTEROV VN \& SOKOLOV EP. 2004. Prediction of stress and hydrodynamic behavior of fluids from multicomponent seismic data. Russian Geology and Geophysics, 45(6): 752-759.

SIBIRYAKOV BP, LEITE LWB \& VIEIRA WWS. 2013. Model of the structured continuum, and the relation between specific surface area, porosity and permeability. Brazilian Journal of Geophysics, 31(4): 559-568.

SIBIRYAKOV BP, LEITE LWB \& VIEIRA WWS. 2015. Behavior of stresses and hydrodynamics from multicomponent seismic data. Brazilian Journal of Geophysics, 33(1): 57-70.

SMIRNOV VI. 1964. Course of higher Mathematics. London: Pergamon Press, $630 \mathrm{pp}$

Recebido em 23 março, 2014 / Aceito em 22 julho, 2014

Received on March 23, 2014 / Accepted on July 22, 2014

\section{NOTES ABOUT THE AUTHORS}

Boris Petrovich Sibiryakov is a Special Professor of Geophysics in the Russian Academy of Sciences (RAS), Novosibirsk Branch, and in the Novosibirsk State University, Russia. At present is a Special Visiting Professor at the Graduate Program in Geophysics of the Universidade Federal do Pará (UFPA), Brazil, participating in the Project Science Without Borders of CAPES/CNPq. Obtained the Masters and Doctorate degrees in Geophysics from the Academy of Sciences in Novosibirsk, Russia. Develops research in applied seismic of fractured media for oil and gas exploration.

Lourenildo Williame Barbosa Leite is a Professor of Geophysics in the Undergraduate and Graduate Courses of the Universidade Federal do Pará (UFPA), Brazil. Obtained the Masters and Doctorate degrees in Geophysics from Saint Louis University, Missouri, USA. Develops research work in applied seismic for oil and gas exploration aiming at velocity analysis, stack, inversion, migration, and attribute analysis. Member of SBGf, SEG, EAGE and SSA societies.

Egor Petrovich Sibiryakov is a Special Researcher of Geophysics in the Russian Academy of Sciences (RAS), Novosibirsk Branch, and in the Novosibirsk State University. At present participates in the Project Science Without Borders of CAPES/CNPq as a Collaborator. Obtained the Masters and Doctorate titles in Geophysics from the Academy of Sciences in Novosibirsk, Russia. Develops research in applied seismic of fractured media for oil and gas exploration.

Wildney Wallacy da Silva Vieira is a Doctorate student at the Graduate Course in Geophysics of the Universidade Federal do Para (UFPA), Brazil. Obtained the Bachelors in Mathematics (2007), and the Masters in Geophysics (2011), both degrees at UFPA. Develops research work in inversion techniques for seismic applied to oil and gas exploration. Area of interest has been ultimately focused at imaging by a complete processing workflow from data quality control to velocity analysis, inversion and migration. 